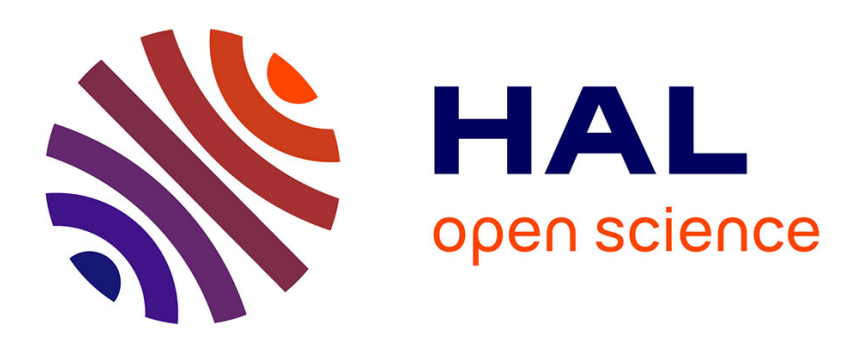

\title{
Fiber-based ultrashort pulse delivery for nonlinear imaging using high-energy solitons
}

S. Saint-Jalm, E. R. Andresen, Patrick Ferrand, A. Bendahmane, Arnaud

Mussot, O. Vanvincq, G. Bouwmans, Alexandre Kudlinski, H. Rigneault

\section{- To cite this version:}

S. Saint-Jalm, E. R. Andresen, Patrick Ferrand, A. Bendahmane, Arnaud Mussot, et al.. Fiber-based ultrashort pulse delivery for nonlinear imaging using high-energy solitons. Journal of Biomedical Optics, 2014, 19 (8), pp.086021. 10.1117/1.JBO.19.8.086021 . hal-01062322

\section{HAL Id: hal-01062322 \\ https://hal.science/hal-01062322}

Submitted on 9 Sep 2014

HAL is a multi-disciplinary open access archive for the deposit and dissemination of scientific research documents, whether they are published or not. The documents may come from teaching and research institutions in France or abroad, or from public or private research centers.
L'archive ouverte pluridisciplinaire HAL, est destinée au dépôt et à la diffusion de documents scientifiques de niveau recherche, publiés ou non, émanant des établissements d'enseignement et de recherche français ou étrangers, des laboratoires publics ou privés. 


\section{Biomedical Optics}

\section{Fiber-based ultrashort pulse delivery for nonlinear imaging using high- energy solitons}

Sarah Saint-Jalm

Esben R. Andresen

Patrick Ferrand

Abdelkrim Bendahmane

Arnaud Mussot

Olivier Vanvincq

Géraud Bouwmans

Alexandre Kudlinski

Hervé Rigneault 


\title{
Fiber-based ultrashort pulse delivery for nonlinear imaging using high-energy solitons
}

\author{
Sarah Saint-Jalm, ${ }^{a}$ Esben R. Andresen, ${ }^{a}$ Patrick Ferrand, ${ }^{a}$ Abdelkrim Bendahmane, ${ }^{b}$ Arnaud Mussot, ${ }^{b}$ \\ Olivier Vanvincq, ${ }^{b}$ Géraud Bouwmans, ${ }^{b}$ Alexandre Kudlinski, ${ }^{b}$ and Hervé Rigneaulta, ${ }^{a, *}$ \\ ${ }^{a}$ Aix Marseille Université, CNRS, Centrale Marseille, Institut Fresnel, UMR 7249, Faculté des Sciences Saint-Jérôme, 13013 Marseille, France \\ bUniversité Lille 1, IRCICA, Laboratoire PhLAM, 59655 Villeneuve d’Ascq, France
}

\begin{abstract}
We present an approach for fiber delivery of femtosecond pulses relying on pulse breakup and soliton self-frequency shift in a custom-made solid-core photonic bandgap fiber. In this scheme, the fiber properties themselves ensure that a powerful Fourier-transform-limited pulse is emitted at the fiber output, hence doing away with the need for complex precompensation and enabling tunability of the excitation. We report highenergy soliton excitation for two-photon fluorescence microspectroscopy over a 100-nm range and multimodal nonlinear imaging on biological samples. ๑ 2014 Society of Photo-Optical Instrumentation Engineers (SPIE) [DOI: 10.1117/1.JBO.19.8 .086021]
\end{abstract}

Keywords: nonlinear imaging; photonics crystal fibers; temporal solitons; ultrafast pulses for imaging.

Paper 140236R received Apr. 14, 2014; revised manuscript received Jul. 16, 2014; accepted for publication Aug. 5, 2014; published online Aug. 26, 2014.

\section{Introduction}

The growing interest in point-scanning microscopy techniques based on nonlinear contrasts such as multiphoton excitation fluorescence, second-harmonic generation (SHG), and thirdharmonic generation or coherent Raman scattering ${ }^{1,2}$ has lead to an increasing need for powerful and versatile light sources. Such a source must generate near-infrared (NIR) ultrashort pulses (typically $\approx 100 \mathrm{fs}$ ) with peak powers high enough to observe signals generated by nonlinear processes taking place in the focal volume. Most of the nonlinear microscopes use bulky optics to relay ultrashort pulses between the femtosecond laser and the microscope stand. Many applications would benefit from fiber delivery of high-energy femtosecond pulses. Such systems would also be very beneficial to the emerging field of local probe or endoscopic nonlinear imaging that aims at miniaturizing point-scanning techniques for sensor and biomedical applications. Although several successful experiments have been conducted over the past 10 years, fiber delivery of Fourier-transform-limited femtosecond pulses remains a challenge today. Various strategies have been explored to address this issue, most of them using Ti:Sapphire lasers for pulse generation in the NIR (800 to $1000 \mathrm{~nm}$ ) and a variety of fibers for pulse propagation and delivery, such as single-mode fibers, ${ }^{3}$ double-clad fibers, ${ }^{4,5}$ fiber bundles, ${ }^{6}$ large-mode area fibers, ${ }^{7}$ double-clad photonic crystal fibers (PCFs), ${ }^{8,9}$ and hollow-core photonic bandgap fibers. ${ }^{10-13}$ Recently, Er- and Yb-doped fiber lasers have been put to use in a new type of all-fiber-optic endoscopes operating at $>1-\mu \mathrm{m}$ wavelength. ${ }^{14,15}$ In most of those examples, the dispersion and nonlinearities of the delivering fibers distort and temporally broaden the pulse. Simultaneous precompensation for dispersion and nonlinearity is complex ${ }^{16,17}$ and only gives a transform-limited output pulse within a narrow input parameter range. Furthermore, none of those techniques allow rapid wavelength tuning of the delivered pulse.

*Address all correspondence to: Hervé Rigneault, E-mail: herve.rigneault@ fresnel.fr
An alternative approach consists of taking advantage of the nonlinearities of a PCF in the anomalous dispersion regime. In this case, fundamental solitons are ejected and red-shifted after pulse breakup and the subsequent soliton self-frequency shift (SSFS) ${ }^{18}$ This way, a femtosecond pulse is generated and delivered by the same fiber. Only fundamental solitons are stable in optical fibers, so the pulse is intrinsically Fourier-transformlimited at the output of the fiber due to the balance between anomalous dispersion and the Kerr nonlinearity responsible for soliton dynamics. Increasing the power at the input of the fiber results in a greater soliton redshift, which opens the door to spectroscopic applications and hyperspectral imaging. This is an elegant way of solving the precompensation problem while enabling tunability. ${ }^{19}$ Solitons of $>1-\mu \mathrm{m}$ wavelengths have been previously used to perform multiphoton microscopy. ${ }^{20-22}$ In this paper, we present results obtained with a custom-made solid-core photonic bandgap (SC-PBG) fiber that has been designed for generating powerful solitons from 800 to $1000 \mathrm{~nm}$, an ideal range for performing microscopy on biological samples. In contrast to previous works in which generation of high-energy solitons in hollow-core photonic bandgap fibers $^{23}$ and higher-order-mode fibers ${ }^{24}$ have been reported, we propose an approach that allows the generation of powerful solitons at input powers compatible with standard light sources available in microscopy labs. We characterize the fiber, demonstrate its use in a spectroscopic scheme, and illustrate its ability to generate two-photon excitation fluorescence (TPEF) and SHG images of biological samples.

\section{Methods}

\subsection{Soliton Dynamics}

The propagation of light in a PCF along its main axis $z$ is governed by the nonlinear Schrödinger equation (NLSE) ${ }^{25}$

0091-3286/2014/\$25.00 @ 2014 SPIE 
$\frac{\partial E(z, t)}{\partial z}=-\frac{i}{2} \beta_{2} \frac{\partial^{2} E(z, t)}{\partial t^{2}}+i \gamma|E(z, t)|^{2} E(z, t)$,

where $\beta_{2}$ is the group velocity dispersion (GVD) and $\gamma$ is the nonlinear parameter. Those two wavelength-dependent parameters characterize the PCF and can be changed only by modifying its design.

An analytical solution to the NLSE can be found in the case of anomalous dispersion $\left(\beta_{2}<0\right)$. This solution describes a pulse of light that propagates without changing its shape, known as a fundamental soliton. It can be expressed as follows:

$E(z, t)=\sqrt{P_{0}} \cdot \operatorname{sech}\left(\frac{t}{T_{0}}\right) \cdot \exp \left(\frac{i\left|\beta_{2}\right| z}{T_{0}^{2}}\right)$,

where $P_{0}$ is the peak power of the soliton and $T_{0}$ is its duration, defined with respect to the full width at half maximum duration by $T_{\text {FWHM }}=2 \ln (1+\sqrt{2}) T_{0}$. Soliton dynamics relies on the interplay between the two processes involved in Eq. (1): the anomalous GVD $\left(\beta_{2}\right)$ and the self-phase modulation created by the nonlinear Kerr effect $(\gamma)$ act against each other in a balanced way, which ensures the preservation of the pulse shape. This relationship is summed up in the following equation, which also links the power and duration of the solitons to each other and to the fiber parameters

$N^{2}=\frac{\gamma P_{0} T_{0}^{2}}{\left|\beta_{2}\right|}$

where $N$ is the soliton order. In this paper, we are only generating fundamental solitons, for which $N=1$, because higherorder solitons are not stable in optical fibers due to their intrinsic higher-order dispersion and/or Raman nonlinearity.

For short pulses $(<5 \mathrm{ps})$, the simplest form of the NLSE [Eq. (1)] is not sufficient to fully describe the behavior of solitons. Higher-order effects have to be taken into account, particularly intrapulse Raman scattering, which is responsible for the SSFS that we will use as a tuning mechanism. When the pulse is very short (typically $\approx 100 \mathrm{fs}$ ), its spectrum is broad enough that an effect similar to stimulated Raman scattering can occur between the blue and the red components, mediated by the vibration modes of silica. Namely, the red part of the spectrum experiences a Raman gain, while the blue part experiences a Raman loss. This results in the continuous redshift of the soliton along its propagation in the fiber. The variation of the central frequency of the soliton is related to the duration of the pulse

$\Delta \omega_{\mathrm{R}} \propto-\frac{\left|\beta_{2}\right| z}{T_{0}^{4}}$.

When more power is injected into the fiber, the duration of the pulse decreases [see Eq. (3)], therefore, the redshift is enhanced. We propose to use this effect as a wavelength tuning mechanism. Indeed, since the redshift is proportional to $T_{0}^{-4}$, a small variation in the pulse duration will cause a large redshift and we can obtain a large tuning range for a virtually constant $T_{0}$.

If the third-order dispersion is small enough, the shape of the pulse remains the same during propagation and it is Fouriertransform-limited at any $z$ in the fiber, which means that it is the shortest pulse allowed by its bandwidth, with a constant time-bandwidth product $\Delta t_{\mathrm{FWHM}} \Delta \nu_{\mathrm{FWHM}}=4[\ln (1+\sqrt{2})]^{2} /$ $\pi^{2} \approx 0.315$ characteristic for $\operatorname{sech}^{2}$ pulses. This is an interesting property for nonlinear microscopy as the contrasts we wish to activate require a high peak power, and therefore a short pulse duration.

\section{$2.2 S C-P B G$ Fiber}

From Eq. (3), we get that the energy of the soliton depends on the dispersion to nonlinear coefficient ratio $\left(\left|\beta_{2}\right| / \gamma\right)$ which are the parameters of the fiber. We can rewrite it in the following way:

$E_{\mathrm{sol}}=\frac{2\left|\beta_{2}\right|}{T_{0} \gamma}$.

In order to get high excitation powers compatible with nonlinear microscopy, one has to fabricate a fiber whose design provides a high $\left|\beta_{2}\right| / \gamma$ ratio. We designed and fabricated an SC-PBG fiber with a doubly periodic cladding consisting of high index inclusions made of germanium-doped silica that are responsible for photonic bandgap guidance and air holes for reducing the confinement losses. Figure 1(a) shows a scanning electron microscope image of the fiber. This fiber resembled the one in Ref. 26, although the transmission window was optimized for $800 \mathrm{~nm}$ lasers. In the present SC-PBG fiber, the generation and redshift of solitons are possible in the first PBG, from the zero dispersion wavelength at $750 \mathrm{~nm}$ to the longwavelength edge at $1030 \mathrm{~nm}$. Such a fiber was shown to generate 3 to 5 times higher-energy solitons than standard PCFs. ${ }^{27}$ Figure 1(b) shows the $\left|\beta_{2}\right| / \gamma$ ratio for the fiber we used in comparison to standard values for other PCFs. Coupling efficiencies were about $30 \%$ to $40 \%$ and the soliton generation efficiency, defined as the ratio of the soliton power over the total input power, was about $10 \%$.

\subsection{Optical Setups}

\subsubsection{Characterization of the SC-PBG fiber}

We launched NIR femtosecond pulses $(800 \mathrm{~nm}, 30 \mathrm{fs}, 1 \mathrm{GHz})$ in sections of the fiber of various lengths for characterization at different input powers. The light was coupled into the fiber with an aspheric lens. To get coupling to the fundamental mode, we imaged the exit end of the fiber on a CMOS camera and maximized the intensity of this mode. The input power was controlled by a half-wave plate and a polarizing beam splitter. The resulting solitons were filtered from the residue of the laser light at $800 \mathrm{~nm}$ as well as the blue-shifted dispersive waves by long-pass filters (LP830RU from Semrock, Rochester, New York or FEL850 from Thorlabs, Newton, New Jersey), and some of their properties were measured, such as average power, spectrum (USB2000, Ocean Optics, Dunedin, Florida), and duration through their autocorrelation trace (Mini, APE, Berlin, Germany).

\subsubsection{Spectroscopy and microscopy}

We conducted two sets of experiments based on the excitation of the sample by the optical solitons generated in the fiber. First, we performed microspectroscopy on liquid samples, then we did microscopy on several samples, including biological ones.

The solitons were generated by a coupling $800 \mathrm{~nm}$ light from either an $80 \mathrm{MHz}$ (Chameleon, 150 fs from Coherent Inc., Santa 
(a)

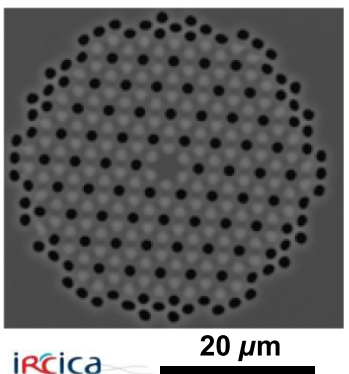

(b)

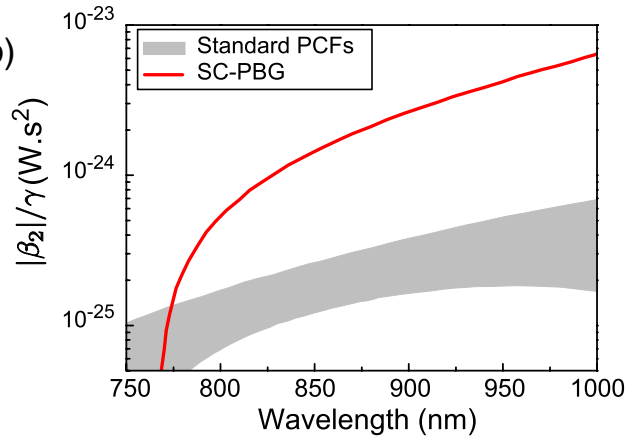

Fig. 1 (a) Scanning electron microscope image of the solid-core photonic bandgap (SC-PBG) fiber. Dark gray: silica; light gray: Ge-doped silica; black: air. (b) Red line: $\left|\beta_{2}\right| / \gamma$ ratios calculated for the SC-PBG fiber used in this work (finite element method simulation). Gray area: typical values of this ratio for standard PCFs.

Clara, California) or a $1 \mathrm{GHz}$ laser (GigaJet, $30 \mathrm{fs}$ from Laser Quantum, Stockport, United Kingdom) into the SC-PBG fiber. As before, the soliton wavelength was controlled by changing the input power, and was filtered from residual and dispersive waves with a long-pass filter to ensure that the soliton was the only excitation pulse reaching the sample. For microspectroscopy, the light was focused on the sample with a long working distance objective $(20 \times, \mathrm{NA}=0.45$ from Olympus, Tokyo, Japan) and collected by another objective $(\mathrm{NA}=0.60)$ in the forward direction. We detected the TPEF on an APD after a set of long-pass and bandpass filters.

For microscopy, we used a high NA objective $(40 \times$, $\mathrm{NA}=1.15$, water immersion from Nikon, Tokyo, Japan) and scanned the focus across the sample with galvanometric mirrors to create TPEF and SHG images in an epi-detection scheme by using two PMTs and an appropriate set of filters and dichroic mirrors.

A scheme representing the two setups combined is shown in Fig. 2.

\section{Results and Discussion}

\subsection{Generation of Solitons in the SC-PBG Fiber}

An example of output spectrum is shown in Fig. 3(a). The redshifted soliton is perfectly fitted by a squared hyperbolic-secant function (red dotted line). The inset of Fig. 3(a) shows the

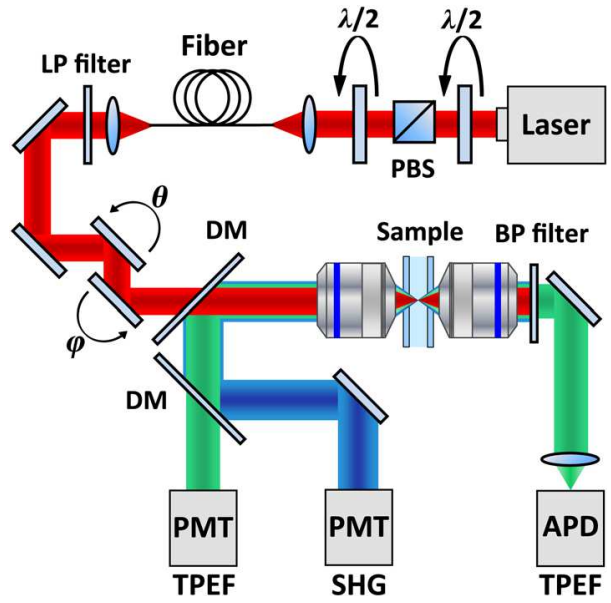

Fig. 2 Scheme of the microspectroscopy and microscopy setup. PBS: polarizing beam splitter; $\lambda / 2$ : half-wave plate; DM: dichroic mirror; LP/BP filter: long-pass/bandpass filter; PMT: photomultiplier tube; and APD: avalanche photodiode. autocorrelation trace for the $900 \mathrm{~nm}$ soliton corresponding to the spectrum. For $\operatorname{sech}^{2}$ pulses, we divide the width FWHM of the autocorrelation trace by 1.54 in order to recover the pulse duration, which in this case was found to be 90 fs. The Fourier-transform-limited duration calculated from the Fourier transform of the spectrum $(\Delta \lambda=10.7 \mathrm{~nm})$ is $80 \mathrm{fs}$. The difference can be explained by the dispersion of the optics between the fiber and the autocorrelator (lens, filter, halfwave plate), estimated to be about $1200 \mathrm{fs}^{2}$. Thus we verify that the soliton is Fourier-transform-limited at the output of the fiber.

Figure 3(b) shows the measured energy of the solitons through the whole tunability window. For each measured point in Fig. 3(b), we calculate the effective $\left|\beta_{2}\right| / \gamma$ ratio with Eq. (3), using duration values calculated from the Fourier transform of the measured spectrum. Figure 3(c) shows those experimental values along with the theoretical curve from finite element method calculations.

We find a good agreement between the simulation and the experiment for fibers up to $10 \mathrm{~m}$, whereas in longer fibers, the calculated $\left|\beta_{2}\right| / \gamma$ ratios are lower than the theory. In a similar fashion, the highest redshift accessible via SSFS increases with fiber length, but it reaches a maximum value at $L=10 \mathrm{~m}$ [see Fig. 3(d)].

In fibers longer than $15 \mathrm{~m}$, several mechanisms can explain the lower values of the effective $\left|\beta_{2}\right| / \gamma$ and the end of the redshift. First, the third-order dispersion arising when the soliton gets closer to the PBG edge decelerates the SSFS in the fiber. ${ }^{28}$ Second, the attenuation experienced by the pulse when it propagates increases the nonlinear length $L_{\mathrm{NL}}=1 /\left(\gamma P_{0}\right)$ which is the characteristic length needed for the soliton to adapt to any changes. At a certain point, the soliton can no longer follow the changes in $P_{0}$, ceases to redshift, and temporally broadens under dispersion. In the end, the redshifted pulse loses its soliton nature during propagation, and Eq. (3) is no longer valid. Figure 3(d) can be interpreted as the redshift versus the fiber length. In view of the above explanation, we can say that soliton nature of the pulse is retained until the plateau.

Finally, we achieve a $100 \mathrm{~nm}$ tuning range for the soliton. The length of the fiber must be less than $10 \mathrm{~m}$ so that Eq. (3) is still valid and the output pulse is Fourier-transform-limited. Then, the fiber length should be chosen according to the tunability and power requirements for the application. In the experiments described in the following, we used the 10-m fiber for spectroscopic application because it displays the best tradeoff between tunability and soliton power, and the 3-m fiber for microscopy to get high soliton power. 


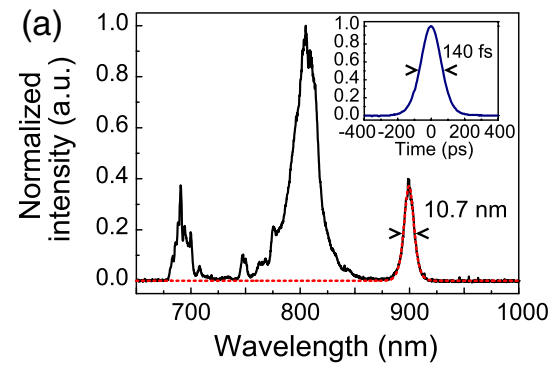

(b)
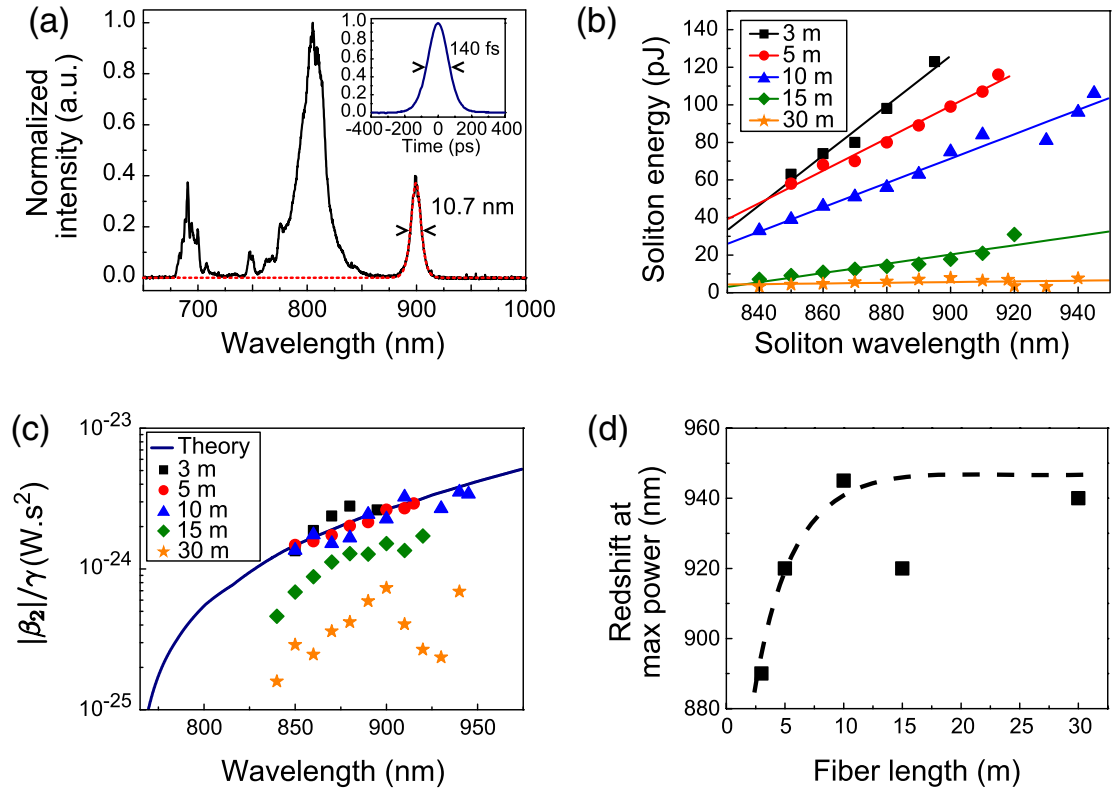

Fig. 3 (a) Black line: spectrum of the output of the fiber. Red dotted line: squared hyperbolic-secant fit of the soliton. Inset: autocorrelation trace of the $900 \mathrm{~nm}$ soliton. (b) Wavelength dependence of the energy of the soliton for fiber lengths from 3 to $30 \mathrm{~m}$. The full lines are linear fits of the experimental points for each fiber. Fiber input average powers were (at $1 \mathrm{GHz}): 420$ to $1400 \mathrm{~mW}(3 \mathrm{~m}) ; 420$ to $1360 \mathrm{~mW}(5 \mathrm{~m})$; 200 to $1380 \mathrm{~mW}(10 \mathrm{~m}) ; 250$ to $1300 \mathrm{~mW}(15 \mathrm{~m}) ; 200$ to $1400 \mathrm{~mW}(30 \mathrm{~m})$. (c) Theoretical $\left|\beta_{2}\right| / \gamma$ ratio (blue line) from simulation and experimental values of this ratio retrieved from measurement of the power and spectrum of the solitons, for several fiber lengths and soliton wavelengths. (d) Redshift of the soliton at maximum input power for varying fiber lengths. The dashed line is a guide to the eye.

\subsection{Microspectroscopy}

We investigated the TPEF response of an aqueous solution of rhodamine $6 \mathrm{G}$ with the setup described above. We first compared the fluorescent levels and the signal-to-noise ratios in the cases of the excitation by solitons generated in the fiber at $80 \mathrm{MHz}$ and at $1 \mathrm{GHz}$.

The TPEF signal at a given wavelength depends on pulse power, duration, and repetition rate $f_{\text {rep }}$ in the following way: ${ }^{29}$

$\langle F\rangle \propto \frac{P_{\mathrm{av}}^{2}}{f_{\text {rep }} \cdot T_{0}}=4 P_{0}^{2} \cdot T_{0} \cdot f_{\text {rep }}$,

where $P_{\mathrm{av}}$ is the average power and $P_{0}$ is the peak power. The energy and duration of one soliton at a given wavelength are fixed by the fiber parameters. Consequently, the only way to significantly increase the TPEF signal in this scheme is to increase the repetition rate.

More specifically, we expect the fluorescence signal to scale linearly with the repetition rate. If the TPEF photon count $S_{1 \text { sol }}$ arising from excitation with a single soliton has mean $\left\langle S_{1 \text { sol }}\right\rangle$ and standard deviation $\sigma_{1 \text { sol }}$, then it follows from the properties of the standard deviation that the TPEF count rate arising from a train of solitons at repetition rate $f_{\text {rep }}$ has mean $\langle S\rangle \propto f_{\text {rep }}\left\langle S_{1 \text { sol }}\right\rangle$ and standard deviation $\sigma \propto \sqrt{f_{\text {rep }}} \sigma_{1 \text { sol }}$. We can then expect both the noise and the signal-to-noise ratio to scale with the square root of the repetition rate.

We measure a 10-fold increase of the fluorescence counts and a 4-fold increase of the signal-to-noise ratio under a 12.5-fold increase of the repetition rate (from $80 \mathrm{MHz}$ to $1 \mathrm{GHz}$ ), as shown in the inset of Fig. 4. The experimental data seem to generally follow the trend predicted by the above calculation. This indicates that we observe only limited saturation effects in the sample when increasing the repetition rate.

The main plot in Fig. 4 shows the TPEF spectra of the same rhodamine $6 \mathrm{G}$ solution. The two spectra (blue circles and green triangles) obtained with excitation by solitons at $80 \mathrm{MHz}$ and $1 \mathrm{GHz}$ were normalized with respect to their average power and repetition rate, taking into account the small saturation effect previously measured. The agreement between the two

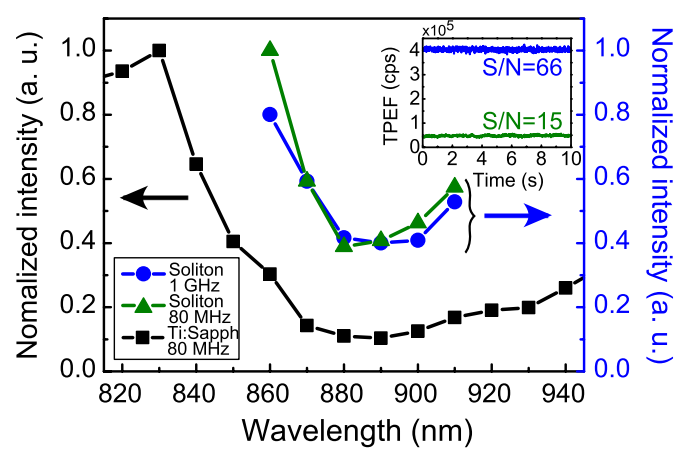

Fig. 4 Two-photon excitation fluorescence (TPEF) spectra of a $170 \mu \mathrm{M}$ solution of rhodamine $6 \mathrm{G}$ in water. Black squares: reference spectrum, measured by exciting the sample with the tunable fs light directly coming from the $80 \mathrm{MHz}$ Ti:Sapphire laser (without passing through the fiber). Blue circles: spectrum acquired under excitation with solitons generated in a $10 \mathrm{~m}$ SC-PBG fiber pumped with an $800 \mathrm{~nm}, 1 \mathrm{GHz}$ laser. Green triangles: spectrum acquired under excitation with solitons generated in a $10 \mathrm{~m}$ SC-PBG fiber pumped with an $800 \mathrm{~nm}, 80 \mathrm{MHz}$ laser. Inset: TPEF traces under excitation with $870 \mathrm{~nm}$ solitons at $1 \mathrm{GHz}$ (blue line) and $80 \mathrm{MHz}$ (green line), with the corresponding signal-to-noise ratios (in $\mathrm{cps}^{1 / 2}$ ). Powers at the focus: black curve: $15 \mathrm{~mW}$; blue: $5 \mathrm{~mW}$; and green: $2 \mathrm{~mW}$. 

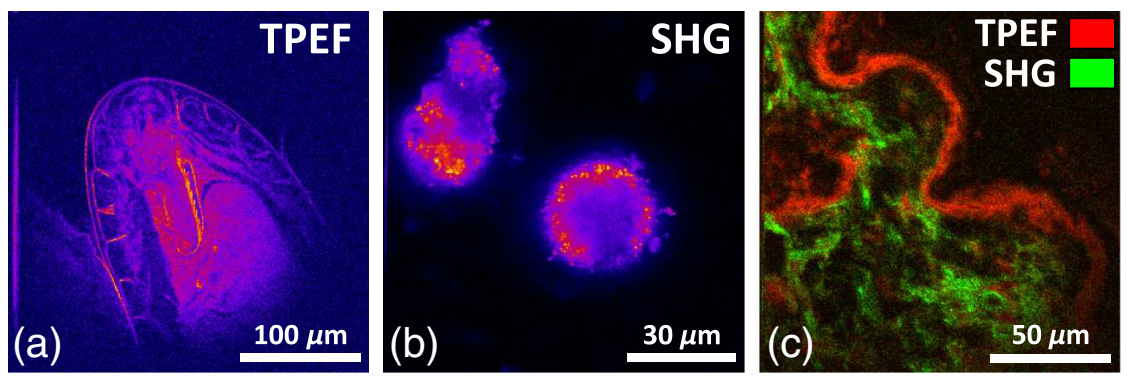

Fig. $5300 \times 300$ pixels TPEF and second-harmonic generation (SHG) images with 10 accumulations each. (a) TPEF image of a fixed Drosophila embryo obtained with an $870 \mathrm{~nm}$ soliton excitation and a $50 \mu$ s dwell time. (b) SHG image of $\mathrm{BaTiO}_{3}$ crystals obtained with an $850 \mathrm{~nm}$ soliton excitation and a $70 \mu \mathrm{s}$ dwell time. (c) Composite image of fixed mouse tumor skin tissue obtained with an $870 \mathrm{~nm}$ soliton excitation and a $70 \mu \mathrm{s}$ dwell time. TPEF is shown in red and SHG in green. Powers in the sample plane: (a) $40 \mathrm{~mW}$; (b) $50 \mathrm{~mW}$; and (c) $55 \mathrm{~mW}$.

spectra is very good, proving the reliability of the soliton as a light source with only a little influence on the parameters of the pumping laser (spectrum, duration, chirp). Another spectrum was used as a control, using the fs pulse directly coming from the $80 \mathrm{MHz}$ Ti:Sapphire laser (black squares) for excitation. The spectra show a good qualitative agreement. Our measurements are also consistent with the two-photon absorption cross section values for rhodamine $6 \mathrm{G}$ found in the literature. ${ }^{29,30}$ However, no quantitative information can be retrieved from the comparison between this spectrum and two first spectra because many parameters vary (spectral width, pulse duration, beam shape).

\subsection{Microscopy}

To demonstrate the imaging capabilities of our system, we performed images of several samples, including biological ones (see Fig. 5). We generated the solitons at $1 \mathrm{GHz}$ repetition rate in a $3 \mathrm{~m}$ fiber to operate at high excitation power and low redshift ( 850 to $890 \mathrm{~nm}$ ). We first tested the two epi-detection channels separately to obtain images of samples known for their strong TPEF and SHG signals, respectively. Figure 5(a) shows a TPEF image of a fixed Drosophila embryo, where the formaldehyde fixation process is responsible for inducing the fluorescence signal. Figure 5(b) shows the SHG image of $\mathrm{BaTiO}_{3}$ crystals. In both cases, the 50 to $70 \mu$ s dwell times that were used are comparable with the ones typically used in nonlinear microscopy. The multiphoton signal is about 10 times lower than images with standard Ti:Sapphire excitation. We have identified the reason for this discrepancy to be the spatial profile of the soliton that is singly peaked but not Gaussian. The microscope we used was not set up to compensate for this effect, which results in a bias against the soliton in this context. We then used the two channels to perform a multimodal image of a biological sample. Figure 5(c) shows a composite TPEF/ SHG image of fixed mouse tumor skin tissue showing structural features that are not distinguishable in white light microscopy. The SHG emitting structures can be identified as collagen fibers, whereas TPEF comes from the stratum corneum.

\section{Conclusions}

We demonstrate the use in microspectroscopy and microscopy of a light source relying on soliton generation and redshift in an SC-PBG fiber. The fiber design was optimized to allow the generation of high-energy solitons, and the laws of soliton dynamics ensured a Fourier-transform-limited pulse at the exit end of the fiber, with no need for precompensation for dispersion. For those reasons, the soliton excitation scheme is efficient for the generation of observable nonlinear signals (such as TPEF and SHG) in the focal volume. Furthermore, the power-dependent redshift of the soliton provides a $100 \mathrm{~nm}$ tunability range, opening the door to spectroscopic applications, hyperspectral imaging, as well as to vibrational spectroscopy and microscopy relying on coherent Raman processes. The speed of wavelength tuning is only limited by the capabilities of the devices used to change the power at the input of the fiber. For example, with a Pockels cell, one could get wavelength switching frequencies up to few MHz. In the current configuration, the wavelength tuning is repeatable enough to operate in an automated fashion over the course of a set of experiments (typically a few hours). This work emphasizes the advantages of increasing the repetition rate in terms of signal level and signal-to-noise ratio. Previous studies also suggest that photodamage associated with multiphoton excitation fluorescence decreases when the repetition rate increases while keeping a constant signal rate, ${ }^{31}$ which is another point in favor of our $1 \mathrm{GHz}$ system for soliton imaging applications of biological samples.

\section{Acknowledgments}

The authors acknowledge financial support from the Centre National de la Recherche Scientifique (CNRS), Aix-Marseille University A*Midex (No. ANR-11-IDEX-0001-02), the Weizmann NaBi European Associated Laboratory, the Ministry of Higher Education and Research, Nord-Pas de Calais Regional Council, FEDER through the Contrat de Projets État Région (CPER) 2007-2013, French Agence Nationale de la Recherche (ANR JCJC TOPWAVE), and ANR grants France Bio Imaging (ANR-10-INSB-04-01) and France Life Imaging (ANR-11INSB-0006) infrastructure networks.

\section{References}

1. W. R. Zipfel, R. M. Williams, and W. W. Webb, "Nonlinear magic: multiphoton microscopy in the biosciences," Nat. Biotechnol. 21(11), 1369-1377 (2003).

2. E. E. Hoover and J. A. Squier, "Advances in multiphoton microscopy technology," Nat. Photonics 7(2), 93-101 (2013).

3. D. Bird and M. Gu, "Compact two-photon fluorescence microscope based on a single-mode fiber coupler," Opt. Lett. 27(12), 1031-1033 (2002).

4. M. T. Myaing, D. J. MacDonald, and X. Li, "Fiber-optic scanning twophoton fluorescence endoscope," Opt. Lett. 31(8), 1076-1078 (2006). 
5. D. R. Rivera et al., "Compact and flexible raster scanning multiphoton endoscope capable of imaging unstained tissue," Proc. Natl. Acad. Sci. U.S.A. 108(43), 17598-17603 (2011).

6. W. Göbel et al., "Miniaturized two-photon microscope based on a flexible coherent fiber bundle and a gradient-index lens objective," Opt. Lett. 29(21), 2521-2523 (2004).

7. S. Moon, G. Liu, and Z. Chen, "Multiphoton endoscopy based on a mode-filtered single-mode fiber," Proc. SPIE 7903, 79032P (2011).

8. M. T. Myaing et al., "Enhanced two-photon biosensing with doubleclad photonic crystal fibers," Opt. Lett. 28(14), 1224-1226 (2003).

9. L. Fu, X. Gan, and M. Gu, "Nonlinear optical microscopy based on double-clad photonic crystal fibers," Opt. Express 13(14), 5528-5534 (2005).

10. B. A. Flusberg et al., "In vivo brain imaging using a portable 3.9 gram two-photon fluorescence microendoscope," Opt. Lett. 30(17), 2272-2274 (2005).

11. C. J. Engelbrecht et al., "Ultra-compact fiber-optic two-photon microscope for functional fluorescence imaging in vivo," Opt. Express 16(8), 5556-5564 (2008).

12. S. Brustlein et al., "Double-clad hollow core photonic crystal fiber for coherent Raman endoscope," Opt. Express 19(13), 12562-12568 (2011).

13. D. M. Huland et al., "Three-photon excited fluorescence imaging of unstained tissue using a GRIN lens endoscope," Biomed. Opt. Express 4(5), 652-658 (2013).

14. K. Murari et al., "Compensation-free, all-fiber-optic, two-photon endomicroscopy at $1.55 \mu \mathrm{m}$," Opt. Lett. 36(7), 1299-1301 (2011).

15. G. Liu et al., "Multiphoton microscopy system with a compact fiberbased femtosecond-pulse laser and handheld probe," J. Biophotonics 4(1-2), 34-39 (2011).

16. M. Lelek et al., "Coherent femtosecond pulse shaping for the optimization of a non-linear micro-endoscope," Opt. Express 15(16), 1015410162 (2007).

17. C. Lefort et al., "Pulse compression and fiber delivery of 45 fs Fourier transform limited pulses at 830 nm," Opt. Lett. 36(2), 292-294 (2011).

18. J. P. Gordon, "Theory of the soliton self-frequency shift," Opt. Lett. 11(10), 662-664 (1986).

19. X.-H. Fang et al., "An all-photonic-crystal-fiber wavelength-tunable source of high-energy sub-100 fs pulses," Opt. Commun. 289, 123-126 (2013).
20. G. McConnell and E. Riis, "Photonic crystal fibre enables short-wavelength two-photon laser scanning fluorescence microscopy with fura-2," Phys. Med. Biol. 49(20), 4757-4763 (2004).

21. E. R. Andresen, P. Berto, and H. Rigneault, "Stimulated Raman scattering microscopy by spectral focusing and fiber-generated soliton as Stokes pulse," Opt. Lett. 36(13), 2387-2389 (2011).

22. K. Wang and C. Xu, "Tunable high-energy soliton pulse generation from a large-mode-area fiber and its application to third harmonic generation microscopy," Appl. Phys. Lett. 99(7), 071112 (2011).

23. D. G. Ouzounov et al., "Generation of megawatt optical solitons in hollow-core photonic band-gap fibers," Science 301(5640), 1702-1704 (2003).

24. M. E. Pedersen et al., "Higher-order-mode fiber optimized for energetic soliton propagation,” Opt. Lett. 37(16), 3459-3461 (2012).

25. G. P. Agrawal, Nonlinear Fiber Optics, Academic Press, London, UK (2001).

26. A. Bétourné et al., "Control of supercontinuum generation and soliton self-frequency shift in solid-core photonic bandgap fibers," Opt. Lett. 34(20), 3083-3085 (2009).

27. A. Bendahmane et al., "Solid-core photonic bandgap fiber for the generation of tunable high-energy solitons," in 3rd Workshop on Specialty Optical Fibers and Their Applications, W3.36, Optical Society of America, Washington DC (2013).

28. O. Vanvincq et al., "Extreme deceleration of the soliton self-frequency shift by the third-order dispersion in solid-core photonic bandgap fibers," J. Opt. Soc. Am. B 27(11), 2328-2335 (2010).

29. M. A. Albota, C. Xu, and W. W. Webb, "Two-photon fluorescence excitation cross sections of biomolecular probes from 690 to $960 \mathrm{~nm}$," Appl. Opt. 37(31), 7352-7356 (1998).

30. N. S. Makarov, M. Drobizhev, and A. Rebane, "Two-photon absorption standards in the 550-1600 $\mathrm{nm}$ excitation wavelength range," Opt. Express 16(6), 4029-4047 (2008).

31. N. Ji, J. C. Magee, and E. Betzig, "High-speed, low-photodamage nonlinear imaging using passive pulse splitters," Nat. Methods 5(2), 197-202 (2008).

Biographies of the authors are not available. 